\title{
On the Performance and Complexity of Irregular Variable Length Codes for Near-Capacity Joint Source and Channel Coding
}

\author{
R. G. Maunder, J. Wang, S. X. Ng, L.-L. Yang, and L. Hanzo
}

\begin{abstract}
In this paper we propose a novel Irregular Variable Length Coding (IrVLC) scheme for near-capacity joint source and channel coding. We employ a number of component Variable Length Coding (VLC) codebooks having different coding rates for encoding particular fractions of the input source symbol stream. These fractions may be chosen with the aid of EXtrinsic Information Transfer (EXIT) charts in order to shape the inverted EXIT curve of the IrVLC codec for ensuring that it does not cross the EXIT curve of a serially concatenated channel codec. In this way, an open EXIT chart tunnel may be created even at low $E_{b} / N_{0}$ values that are close to the capacity bound of the channel. We propose iteratively decoded serially concatenated IrVLC designs amalgamated with Trellis Coded Modulation (TCM). These schemes are shown to be capable of operating within $0.6 \mathrm{~dB}$ of the uncorrelated narrowband Rayleigh fading channel's capacity bound using an average interleaver length of $217,500 \mathrm{bits}$ and an effective bandwidth efficiency of $1.56 \mathrm{bit} / \mathrm{s} / \mathrm{Hz}$, assuming ideal Nyquist filtering. By contrast, the equivalentrate regular VLC-based bench-marker schemes were found to be capable of operating at a higher distance of $1.1 \mathrm{~dB}$ from the capacity bound, which is nearly twice that of the proposed IrVLC-TCM scheme. Additionally, an Irregular Convolutional Coding (IrCC) based bench-marker was found to be capable of operating at $0.8 \mathrm{~dB}$ from the capacity bound, owing to its slightly eroded performance when operating with the considered interleaver length.
\end{abstract}

Index Terms - Variable length codes, joint source and channel coding, trellis codes, information rates.

\section{INTRODUCTION}

$\mathbf{I}^{\mathrm{r}}$ RREGULAR Convolutional Coding (IrCC) [1] has been proposed for employment as an outer channel codec in iteratively decoded schemes. This amalgamates a number of component Convolutional Codes (CC) having different coding rates, each of which encodes an appropriately selected fraction of the input bit stream. More specifically, the appropriate fractions may be selected with the aid of EXtrinsic Information Transfer (EXIT) chart analysis [2], in order to shape the inverted EXIT curve of the composite IrCC for ensuring that it does not cross the EXIT curve of the inner codec. In this way, an open EXIT chart tunnel may be created at

Manuscript received November 11, 2006; revised March 6, 2007 and April 26, 2007; accepted June 8, 2007. The associate editor coordinating the review of this paper and approving it for publication was G. Vitetta. The financial support of the EPSRC, Swindon UK and the EU under the auspices of the PHOENIX and NEWCOM projects is gratefully acknowledged.

The authors are with the School of Electrical \& Computer Science, University of Southampton, UK (e-mail: \{rm02r, sxn, 1ly, lh $\} @$ ecs.soton.ac.uk, dr.jin.wang@gmail.com).

Digital Object Identifier 10.1109/TWC.2008.060891. low $E_{b} / N_{0}$ values, which implies approaching the channel's capacity bound [3]. This was demonstrated for the serial concatenation of IrCCs combined with precoded equalisation in [4], for example.

Similarly to binary Bose-Chaudhuri-Hocquenghem $(\mathrm{BCH})$ codecs [5], the constituent binary CCs [5] of an IrCC codec are unable to exploit the unequal source symbol occurrence probabilities that are typically associated with audio, speech, image and video sources [6] [7]. Since the exploitation of all available redundancy is required for near-capacity operation [8], typically a Huffman source encoder [9] is employed for removing this redundancy, before channel encoding commences. However, Huffman decoding is very sensitive to bit errors, requiring the low Bit Error Ratio (BER) reconstruction of the Huffman encoded bits in order that a low Symbol Error Ratio (SER) may be achieved [10].

This motivates the application of the Variable Length Error Correction (VLEC) class of Variable Length Codes (VLCs) [10] as an alternative to the Huffman and BCH or CC coding of sequences of source symbols having values with unequal probabilities of occurrence. In VLEC coding, the source symbols are represented by binary codewords of varying lengths. Typically, the more frequently that a particular source symbol value occurs, the shorter its VLEC codeword is, resulting in a reduced average codeword length $L$. In order that each valid VLEC codeword sequence may be uniquely decoded, a lower bound equal to the source entropy $E$ is imposed upon the average codeword length $L$. Any discrepancy between $L$ and $E$ is quantified by the coding rate $R=E / L \in$ $[0,1]$ and may be attributed to the intentional introduction of redundancy into the VLEC codewords. Naturally, this intentionally introduced redundancy imposes code constraints that limit the set of legitimate sequences of VLEC-encoded bits. Like the code constraints of $\mathrm{CC}$ and $\mathrm{BCH}$ coding [5], the VLC code constraints may be exploited for providing an additional error correcting capability during VLEC decoding [10]. Furthermore, unlike in $\mathrm{CC}$ and $\mathrm{BCH}$ decoding [5], any redundancy owing to the unequal occurrence probabilities of the source symbol values may be additionally exploited during VLEC decoding [10].

Depending on the VLEC coding rate, the associated code constraints render VLEC decoding substantially less sensitive to bit errors than Huffman decoding is. Hence, a coding gain of $1 \mathrm{~dB}$ at an SER of $10^{-5}$ has been observed by employing VLEC coding having a particular coding rate instead of a 
concatenated Huffman and $\mathrm{BCH}$ coding scheme having the same coding rate [10].

This motivates the application of the irregular coding concept to VLEC coding for employment in the near-capacity joint source and channel coding of sequences of source symbols having values with unequal occurrence probabilities. More specifically, we employ a novel Irregular Variable Length Coding (IrVLC) scheme as our outer source codec, which we serially concatenate [11] [12] with an inner channel codec for the sake of exchanging extrinsic information. In analogy to IrCC, the proposed IrVLC scheme employs a number of component VLEC codebooks having different coding rates, which are used for encoding appropriately selected fractions of the input source symbol stream. In this way, the resultant composite inverted EXIT curve may be shaped for ensuring that it does not cross the EXIT curve of the inner channel codec.

The rest of this paper is outlined as follows. In Section II, we propose iteratively decoded schemes, in which we opt for serially concatenating IrVLC with Trellis Coded Modulation (TCM). Furthermore, Section II additionally introduces our bench-mark schemes, where IrVLC is replaced by regular VLCs having the same coding rate. The design and EXIT chart aided characterisation of these schemes is detailed in Section III. In Section IV, we quantify the attainable performance improvements offered by the proposed IrVLC arrangements compared to the regular VLC bench-marker schemes, as provided in [13]. Furthermore in Section IV of this contribution, we additionally consider a Huffman coding and IrCC based bench-marker, as well as presenting significant new results pertaining to the computational complexity of the considered schemes. More specifically, we quantify the computational complexity required for achieving a range of source sample reconstruction qualities at a range of Rayleigh fading channel $E_{b} / N_{0}$ values. Finally, we offer our conclusions in Section V.

\section{OVERVIEW OF Proposed SCHEMES}

In this section we provide an overview of a number of serially concatenated and iteratively decoded joint source and channel coding schemes. Whilst the novel IrVLC scheme introduced in this paper may be tailored for operating in conjunction with any inner channel codec, we opt for employing TCM [14] in each of our considered schemes. This provides error protection without any bandwidth expansion or effective bit-rate reduction by accommodating the additional redundancy by transmitting more bits per channel symbol. The choice of TCM is further justified, since A Posteriori Probability (APP) TCM Soft-In Soft-Out (SISO) decoding, similarly to APP SISO IrVLC decoding, operates on the basis of Add-Compare-Select (ACS) operations within a trellis structure. Hence, the APP SISO IrVLC and TCM decoders can share resources in systolic-array based chips, facilitating a cost effective implementation. Furthermore, we will show that TCM exhibits attractive EXIT characteristics in the proposed IrVLC context even without requiring TTCM- or BICM-style internal iterative decoding [5].

Our considered schemes differ in their choice of the outer source codec. Specifically, we consider a novel IrVLC codec and an equivalent regular VLC-based bench-marker in this role. In both cases we employ both Symbol-Based (SB) [15] [16] and Bit-Based (BB) [17] VLC decoding, resulting in a total of four different configurations. We refer to these four schemes as the SBIrVLC-, BBIrVLC-, SBVLC- and BBVLC-TCM arrangements, as appropriate. A schematic that is common to each of these four considered schemes is provided in Figure 1.

\section{A. Joint source and channel coding}

The schemes considered are designed for facilitating the near-capacity detection of source samples received over an uncorrelated narrowband Rayleigh fading channel. We consider the case of independent identically distributed (i.i.d.) source samples, which may represent the prediction residual error that remains following the predictive coding of audio, speech, image or video information [6] [7], for example. A Gaussian source sample distribution is assumed, since this has widespread applications owing to the wide applicability of the central limit theorem. Note however that with the aid of suitable adaptation, the techniques proposed in this treatise may be just as readily applied to arbitrary source sample distributions.

In the transmitter of Figure 1, the real-valued source samples are quantized to $K$ number of quantization levels in the block $Q$. The resultant frame of quantized source samples is synonymously referred to as the frame of source symbols $\mathbf{s}$ here. Each source symbol in this frame indexes the particular quantization level $\tilde{e}^{k}, k \in[1 \ldots K]$, that represents the corresponding source sample in the frame e with the minimum squared error. Owing to the lossy nature of quantization, distortion is imposed upon the reconstructed source sample frame $\tilde{\mathbf{e}}$ that is obtained by the receiver of Figure 1, following inverse quantization in the block $Q^{-1}$. The total distortion expected depends on both the original source sample distribution as well as on the number of quantization levels $K$. This distortion may be minimised by employing LloydMax quantization [18] [19]. Here, a $K=16$-level LloydMax quantization scheme is employed, which achieves an expected Signal to Quantization Noise Ratio (SQNR) of about $20 \mathrm{~dB}$ for a Gaussian source [18]. Note however that with suitable adaptation, the techniques advocated in this treatise may be just as readily applied to arbitrary quantisers. Also note that Lloyd-Max quantization results in a large variation in the occurrence probabilities of the resultant source symbol values. These occurrence probabilities are given by integrating the source Probability Density Function (PDF) between each pair of adjacent decision boundaries. In the case of our $K=16$-level quantizer, the source symbol values' occurrence probabilities vary by more than an order of magnitude between 0.0082 and 0.1019 . These probabilities correspond to symbol informations spanning between 3.29 and 6.93 bits/symbol, motivating the application of VLC and giving a source entropy of $E=3.77$ bits/symbol.

In the transmitter of the proposed scheme, the LloydMax quantized source symbol frame $\mathbf{s}$ is decomposed into $M=300$ sub-frames $\left\{\mathbf{s}^{\mathbf{m}}\right\}_{m=1}^{M}$, as shown in Figure 1. In the case of the SBIrVLC- and SBVLC-TCM schemes, 


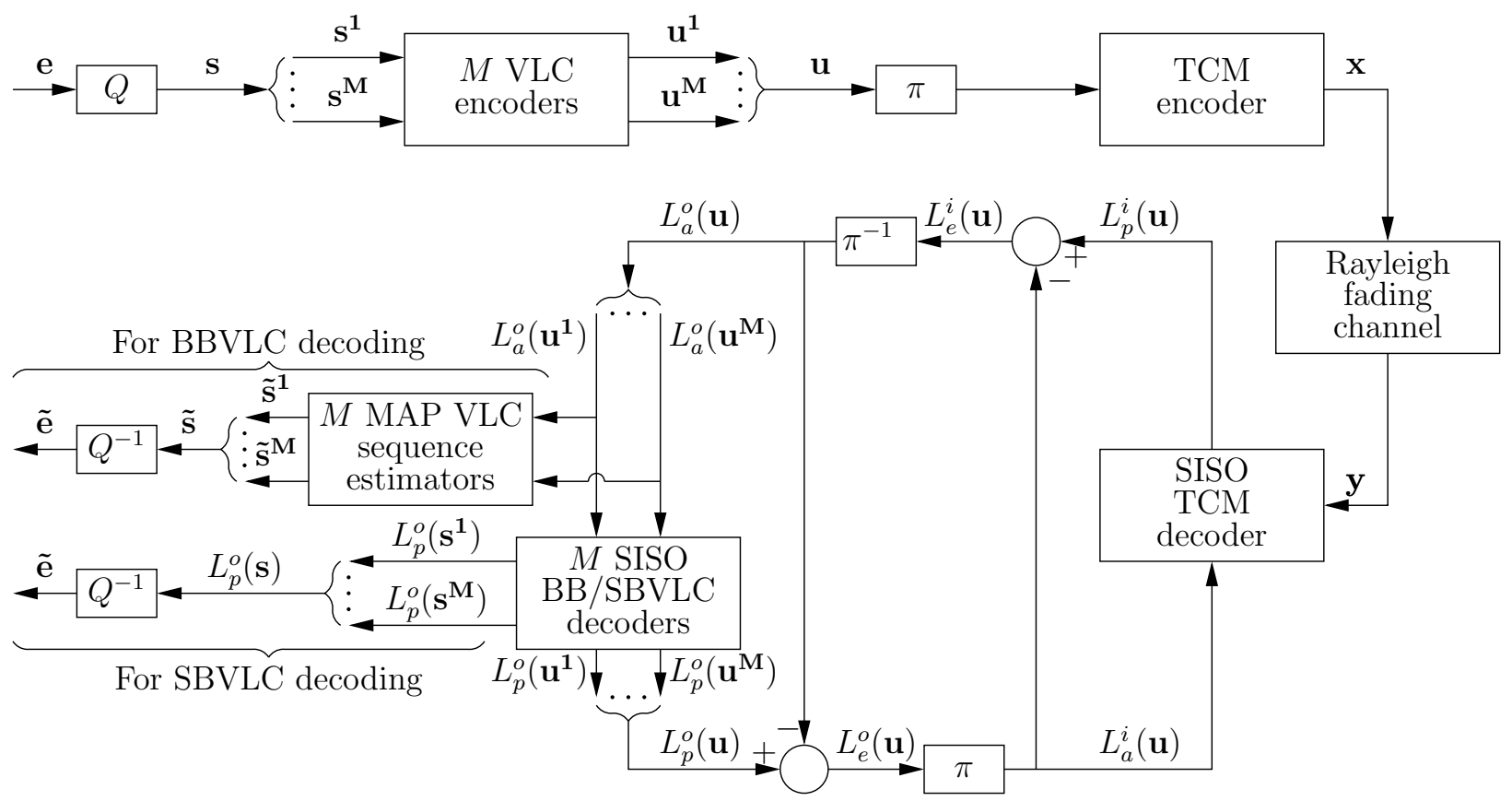

Fig. 1. Schematic of the SBIrVLC-, BBIrVLC-, SBVLC- and BBVLC-TCM schemes. In the IrVLC schemes, the $M$ number of VLC encoders, APP SISO decoders and MAP sequence estimators are each based upon one of $N$ number of VLC codebooks. By contrast, in the VLC bench-markers, all of the $M$ number of VLC encoders, decoders and sequence estimators are based upon the same VLC codebook.

this decomposition is necessary for the sake of limiting the computational complexity of VLC decoding, since the number of transitions in the symbol-based VLC trellis is inversely proportional to the number of sub-frames in this case [15]. We opt for employing the same decomposition of the source symbol frames into sub-frames in the case of the BBIrVLCand BBVLC-TCM schemes for the sake of ensuring that we make a fair comparison with the SBIrVLC- and SBVLC-TCM schemes. This is justified, since the decomposition considered benefits the performance of the BBIrVLC- and BBVLC-TCM schemes, as will be detailed below. Each source symbol subframe $\mathbf{s}^{\mathbf{m}}$ comprises $J=100$ source symbols. Hence, the total number of source symbols in a source symbol frame becomes $M \cdot J=30,000$. As described above, each LloydMax quantized source symbol in the sub-frame $\mathbf{s}^{\mathbf{m}}$ has a $K$ ary value $s_{j}^{m} \in[1 \ldots K]$, where we have $j \in[1 \ldots J]$.

As described in Section I, we employ $N$ number of VLC codebooks to encode the source symbols, where we opted for $N=15$ for the SBIrVLC and BBIrVLC schemes and $N=1$ for the regular SBVLC and BBVLC schemes. Each Lloyd-Max quantized source symbol sub-frame $\mathbf{s}^{\mathbf{m}}$ is VLCencoded using a single VLC codebook VLC ${ }^{n}$, where we have $n \in[1 \ldots N]$. In the case of the SBIrVLC and BBIrVLC schemes, the particular fraction $C^{n}$ of the set of source symbol sub-frames that is encoded by the specific VLC codebook VLC $^{n}$ is fixed and will be derived in Section III. The specific Lloyd-Max quantized source symbols having the value of $k \in[1 \ldots K]$ and encoded by the specific VLC codebook $\mathbf{V L C}^{n}$ are represented by the codeword $\mathbf{V L C}{ }^{n, k}$, which has a length of $I^{n, k}$ bits. The $J=100$ VLC codewords that represent the $J=100$ Lloyd-Max quantized source symbols in each source symbol sub-frame $\mathbf{s}^{\mathbf{m}}$ are concatenated to provide the transmission sub-frame $\mathbf{u}^{\mathbf{m}}=\left\{\mathbf{V L} \mathbf{C}^{n, s_{j}^{m}}\right\}_{j=1}^{J}$.
Owing to the variable lengths of the VLC codewords, each of the $M=300$ transmission sub-frames typically comprises a different number of bits. In order to facilitate the VLC decoding of each transmission sub-frame $\mathbf{u}^{\mathbf{m}}$, it is necessary to explicitly convey its length $I^{m}=\sum_{j=1}^{J} I^{n, s_{j}^{m}}$ to the receiver. Furthermore, this highly error sensitive side information must be reliably protected against transmission errors. This may be achieved using a low rate block code, for example. For the sake of avoiding obfuscation, this is not shown in Figure 1. Note that the choice of the specific number of sub-frames $M$ in each frame constitutes a trade-off between the computational complexity of SBVLC decoding or the performance of BBVLC decoding and the amount of side information that must be conveyed. In Section III, we shall comment on the amount of side information that is required for reliably conveying the specific number of bits in each transmission sub-frame to the decoder.

In the scheme's transmitter, the $M=300$ number of transmission sub-frames $\left\{\mathbf{u}^{\mathbf{m}}\right\}_{m=1}^{M}$ are concatenated. As shown in Figure 1, the resultant transmission frame $\mathbf{u}$ has a length of $\sum_{m=1}^{M} I^{m}$ bits.

In the proposed scheme, the VLC codec is protected by a serially concatenated TCM codec. Following VLC encoding, the bits of the transmission frame $\mathbf{u}$ are interleaved in the block $\pi$ of Figure 1 and TCM encoded in order to obtain the channel's input symbols $\mathbf{x}$, as shown in Figure 1. These are transmitted over an uncorrelated narrowband Rayleigh fading channel and are received as the channel's output symbols $\mathbf{y}$, as seen in Figure 1.

\section{B. Iterative decoding}

In the receiver, APP SISO TCM- and VLC-decoding are performed iteratively, as shown in Figure 1. Both of these 
decoders invoke the Bahl-Cocke-Jelinek-Raviv (BCJR) algorithm [20] on the basis of their trellises. Symbol-based trellises are employed in the case of TCM [14], SBIrVLC and SBVLC [15] [16] decoding, whilst BBIrVLC and BBVLC decoding rely on bit-based trellises [17]. All BCJR calculations are performed in the logarithmic probability domain and using an eight-entry lookup table for correcting the Jacobian approximation in the Log-MAP algorithm [5]. The proposed approach requires only Add, Compare and Select (ACS) computational operations during iterative decoding, which will be used as our complexity measure, since it is characteristic of the complexity/area/speed trade-offs in systolic-array based chips.

As usual, extrinsic soft information, represented in the form of Logarithmic Likelihood Ratios (LLRs) [21], is iteratively exchanged between the TCM and VLC decoding stages for the sake of assisting each other's operation, as detailed in [11] and [12]. In Figure 1, $L(\cdot)$ denotes the LLRs of the bits concerned (or the log-APPs of the specific symbols as appropriate), where the superscript $i$ indicates inner TCM decoding, while $o$ corresponds to outer VLC decoding. Additionally, a subscript denotes the dedicated role of the LLRs (or log-APPs), with $a, p$ and $e$ indicating a priori, a posteriori and extrinsic information, respectively.

Just as $M=300$ separate VLC encoding processes are employed in the proposed scheme's transmitter, $M=300$ separate VLC decoding processes are employed in its receiver. In parallel to the composition of the bit-based transmission frame $\mathbf{u}$ from its $M=300$ sub-frames, the a priori LLRs $L_{a}^{o}(\mathbf{u})$ are decomposed into $M=300$ sub-frames, as shown in Figure 1. This is achieved with the aid of the explicit side information that conveys the number of bits $I^{m}$ in each transmission sub-frame $\mathbf{u}^{\mathbf{m}}$. Each of the $M=300$ VLC decoding processes is provided with the a priori LLR subframe $L_{a}^{o}\left(\mathbf{u}^{\mathbf{m}}\right)$ and in response it generates the a posteriori LLR sub-frame $L_{p}^{o}\left(\mathbf{u}^{\mathbf{m}}\right), m \in[1 \ldots M]$. These a posteriori LLR sub-frames are concatenated in order to provide the $a$ posteriori LLR frame $L_{p}^{o}(\mathbf{u})$, as shown in Figure 1 .

In the case of SBIrVLC and SBVLC decoding, each of the $M=300$ VLC decoding processes additionally provides log-APPs pertaining to the corresponding source symbol subframe $L_{p}^{o}\left(\mathbf{s}^{\mathbf{m}}\right)$. This comprises a set of $K$ number of logAPPs for each source symbol $s_{j}^{m}$ in the sub-frame $\mathbf{s}^{\mathbf{m}}$, where $j \in[1 \ldots J]$. Each of these log-APPs provides the logarithmic probability that the corresponding source symbol $s_{j}^{m}$ has the particular value $k \in[1 \ldots K]$. In the receiver of Figure 1, the source symbols' log-APP sub-frames are concatenated to provide the source symbol log-APP frame $L_{p}^{o}(\mathbf{s})$. By inverse-quantising this soft information in the block $Q^{-1}$, a frame of Minimum Mean Squared Error (MMSE) source sample estimates ẽ can be obtained. More specifically, each reconstructed source sample is obtained by using the corresponding set of $K$ source symbol value probabilities to find the weighted average of the $K$ number of quantization levels $\left\{\tilde{e}^{k}\right\}_{k=1}^{K}$.

Conversely, in the case of BBIrVLC and BBVLC decoding, no symbol-based a posteriori output is available. In this case, each source symbol sub-frame $\mathbf{s}^{\mathbf{m}}$ is estimated from the corresponding a priori LLR sub-frame $L_{a}^{o}\left(\mathbf{u}^{\mathbf{m}}\right)$. This may be achieved by employing Maximum A Posteriori (MAP) sequence estimation operating on a bit-based trellis structure, as shown in Figure 1. Unlike in APP SISO SBIrVLC and SBVLC decoding, bit-based MAP sequence estimation cannot exploit the knowledge that each sub-frame $\mathbf{s}^{\mathbf{m}}$ comprises $J=100$ source symbols. For this reason, the resultant hard decision estimate $\tilde{\mathbf{s}}^{\mathrm{m}}$ of each source symbol sub-frame $\mathbf{s}^{\mathrm{m}}$ may or may not contain $J=100$ source symbols. In order that we may prevent the loss of synchronisation that this would imply, source symbol estimates are removed from, or appended to the end of each source symbol sub-frame estimate $\tilde{\mathbf{s}}^{\mathrm{m}}$ for ensuring that they each comprise exactly $J=100$ source symbol estimates. Note that it is the decomposition of the source symbol frame $\mathbf{s}$ into sub-frames that provides this opportunity to mitigate the loss of synchronisation that is associated with bit-based MAP VLC sequence estimation. Hence the decomposition of the source symbol frame $\mathrm{s}$ into sub-frames benefits the performance of the BBIrVLC- and BBVLC-TCM schemes, as mentioned above.

Following MAP sequence estimation, the adjusted source symbol sub-frame estimates $\tilde{\mathbf{s}}^{\mathrm{m}}$ are concatenated for the sake of obtaining the source symbol frame estimate $\tilde{\mathbf{s}}$. This may be inverse-quantised in order to obtain the source sample

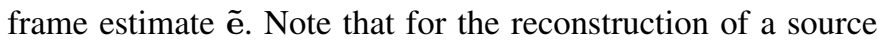
sample frame estimate $\tilde{\mathbf{e}}$ from a given a priori LLR frame $L_{a}^{o}(\mathbf{u})$, a higher level of source distortion may be expected in the BBIrVLC- and BBVLC-TCM schemes than in the corresponding SBIrVLC- and SBVLC-TCM schemes. This is due to the BBIrVLC- and BBVLC-TCM schemes' reliance on hard decisions as opposed to the soft decisions of the SBIrVLC- and SBVLC-TCM schemes. However, this reduced performance substantially benefits us in terms of a reduced complexity, since the bit-based VLC decoding trellis employed during APP SISO BBIrVLC and BBVLC decoding and MAP sequence estimation contains significantly less transitions than the symbol-based VLC decoding trellis of APP SISO SBIrVLC and SBVLC decoding.

In the next section we detail the design of our IrVLC scheme and characterise each of the SBIrVLC-, BBIrVLC-, SBVLC- and BBVLC-TCM schemes with the aid of EXIT chart analysis.

\section{Parameter Design for the Proposed Schemes}

\section{A. Scheme hypothesis and parameters}

As described in Section I, the SBIrVLC and BBIrVLC schemes may be constructed by employing a number of VLC codebooks having different coding rates, each of which encodes an appropriately chosen fraction of the input source symbols. We opted for using $N=15$ VLC codebooks VLC $^{n}$, $n \in[1 \ldots N]$, that were specifically designed for encoding $K=16$-level Lloyd-Max quantized Gaussian i.i.d. source samples. These 15 VLC codebooks were selected from a large number of candidate codebooks in order to provide a suite of similarly-spaced EXIT curves. More specifically, the $N=15$ VLC codebooks comprised 13 Variable Length Error Correcting (VLEC) designs having various so-called minimum block-, convergence- and divergence-distances as defined in [22], complemented by a symmetric- and an asymmetric- 
Reversible Variable Length Coding (RVLC) design [22]. In all codebooks, a free distance of at least $d_{f}=2$ was employed, since this supports convergence to an infinitesimally low BER [23]. The resultant average VLC codeword lengths of $L^{n}=\sum_{k=1}^{K} P(k) \cdot I^{n, k}, n \in[1 \ldots N]$, were found to range from 3.94 to 12.18 bits/symbol. When compared to the source symbol entropy of $E=-\sum_{k=1}^{K} P(k) \cdot \log _{2}(P(k))=3.77$ bits/symbol, these correspond to coding rates of $R^{n}=E / L^{n}$ spanning the range of 0.31 to 0.96 .

As will be detailed below, our SBIrVLC and BBIrVLC schemes were designed under the constraint that they have an overall coding rate of $R=0.52$. This value was chosen, since it is the coding rate of the VLC codebook $\mathrm{VLC}^{10}$, which we employ in our SBVLC and BBVLC bench-markers using $N=1$ codebook. This coding rate results in an average interleaver length of $M \cdot J \cdot E / R=217,500$ bits for all the schemes considered.

In-phase Quadrature-phase (IQ)-interleaved TCM having eight trellis-states per symbol along with 3/4-rate coded 16Level Quadrature Amplitude Modulation (16QAM) is employed, since this is appropriate for transmission over uncorrelated narrowband Rayleigh fading channels. Ignoring the modest bitrate contribution of conveying the side information, the bandwidth efficiency of the schemes considered is therefore $\eta=0.52 \cdot 0.75 \cdot \log _{2}(16)=1.56 \mathrm{bit} / \mathrm{s} / \mathrm{Hz}$, assuming ideal Nyquist filtering having a zero excess bandwidth. This value corresponds to the channel capacity of the uncorrelated narrowband Rayleigh fading channel at an $E_{b} / N_{0}$ value of $2.6 \mathrm{~dB}$ [24]. Given this point on the corresponding channel capacity curve, we will be able to quantify, how closely the proposed schemes may approach this ultimate limit.

Recall from Section II that it is necessary to convey the length of each transmission sub-frame $\mathbf{u}^{\mathbf{m}}$ to the receiver in order to facilitate its VLC decoding. It was found for all considered schemes that a single 10-bit fixed-length codeword of side information is sufficient for conveying the length of each of the $M=300$ transmission sub-frames $\mathbf{u}^{\mathbf{m}}$ in each transmission frame $\mathbf{u}$. As suggested in Section II, this error sensitive side information may be protected by a low-rate block code in order to ensure its reliable transmission. Note that since even a $1 / 3$-rate repetition code encodes the side information with only about $4 \%$ of the expected transmission frame length, we consider the overhead of conveying side information to be acceptable.

\section{B. EXIT chart analysis and optimization}

We now consider the EXIT characteristics of the various components of our various schemes. In all cases, EXIT curves were generated using uncorrelated Gaussian distributed $a$ priori LLRs with the assumption that the transmission frame's bits have equiprobable values. This is justified, because we employ a long interleaver and because the entropy of the VLC encoded bits was found to be at least 0.99 for all considered VLC codebooks. All mutual information measurements were made using the histogram-based approximation of the LLR PDFs [2].

In Figure 2, we provide the EXIT curves $I_{e}^{i}\left(I_{a}^{i}, E_{b} / N_{0}\right)$ of the TCM scheme for a number of $E_{b} / N_{0}$ values above the

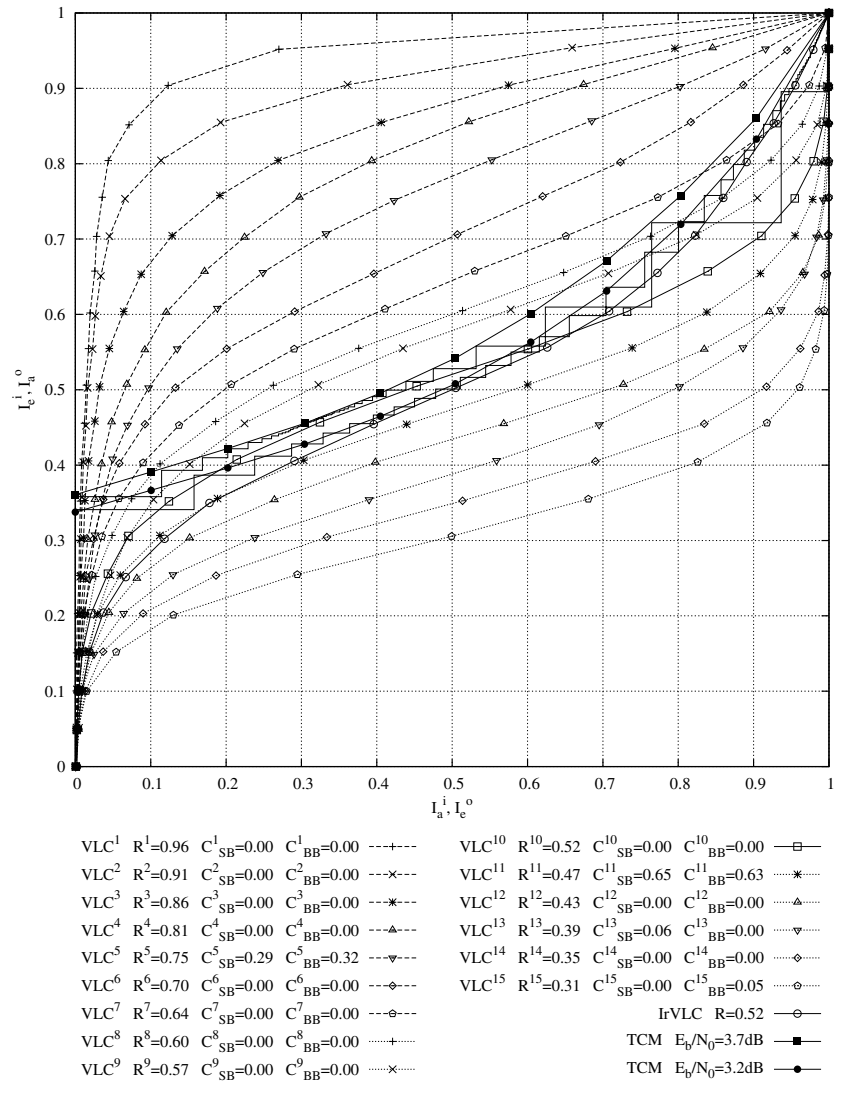

Fig. 2. Inverted VLC EXIT curves and TCM EXIT curves.

channel capacity bound of $2.6 \mathrm{~dB}$. The inverted EXIT curves $I_{a}^{o, n}\left(I_{e}^{o}\right)$ plotted for the $N=15$ VLC codebooks, together with their coding rates $R^{n}$, are also given in Figure 2. Note that these curves were obtained using bit-based VLC decoding, but very similar curves may be obtained for symbol-based decoding.

The inverted EXIT curve of an IrVLC scheme $I_{a}^{o}\left(I_{e}^{o}\right)$ can be obtained as the appropriately weighted superposition of the $N=15$ component VLC codebooks' EXIT curves,

$$
I_{a}^{o}\left(I_{e}^{o}\right)=\sum_{n=1}^{N} \alpha^{n} I_{a}^{o, n}\left(I_{e}^{o}\right)
$$

where $\alpha^{n}$ is the fraction of the transmission frame $\mathbf{u}$ that is generated by the specific component codebook $\mathbf{V L C}^{n}$. Note that the values of $\alpha^{n}$ are subject to the constraints

$$
\sum_{n=1}^{N} \alpha^{n}=1, \quad \alpha^{n} \geq 0 \forall n \in[1 \ldots N] .
$$

The specific fraction of source symbol sub-frames $\mathbf{s}^{\mathbf{m}}$ that should be encoded by the specific component codebook VLC $^{n}$ in order that it generates a fraction $\alpha^{n}$ of the transmission frame $\mathbf{u}$, is given by

$$
C^{n}=\alpha^{n} \cdot R^{n} / R,
$$

where $R=0.52$ is the desired overall coding rate. Again, the 
specific values of $C^{n}$ are subject to the constraints

$$
\sum_{n=1}^{N} C^{n}=\sum_{n=1}^{N} \alpha^{n} \cdot R^{n} / R=1, \quad C^{n} \geq 0 \forall n \in[1 \ldots N] .
$$

Beneficial values of $C^{n}$ may be chosen by ensuring that there is an open EXIT tunnel between the inverted IrVLC EXIT curve and the EXIT curve of TCM at an $E_{b} / N_{0}$ value that is close to the channel capacity bound. This may be achieved using the iterative EXIT-chart matching process of [1] to adjust the values of $\left\{C^{n}\right\}_{n=1}^{N}$ under the constraints of (2) and (4) for the sake of minimising the error function

$$
\left\{C^{n}\right\}_{n=1}^{N}=\underset{\left\{C^{n}\right\}_{n=1}^{N}}{\operatorname{argmin}}\left(\int_{0}^{1} e(I)^{2} \mathrm{~d} I\right)
$$

where

$$
e(I)=I_{e}^{i}\left(I, E_{b} / N_{0}\right)-I_{a}^{o}(I)
$$

is the difference between the inverted IrVLC EXIT curve and the EXIT curve of TCM at a particular target $E_{b} / N_{0}$ value. Note that in order to ensure that the design results in an open EXIT tunnel, we must impose the additional constraint of

$$
e(I)>0 \forall I \in[0,1] \text {. }
$$

Open EXIT tunnels were found to be achievable for both the SBIrVLC- and the BBIrVLC-TCM schemes at an $E_{b} / N_{0}$ of $3.2 \mathrm{~dB}$, which is just $0.6 \mathrm{~dB}$ from the channel capacity bound of $2.6 \mathrm{~dB}$. The inverted BBIrVLC EXIT curve is shown in Figure 2, which is similar to the SBIrVLC EXIT curve not shown here for reasons of space-economy. The corresponding values of $C^{n}$ are provided for both the SBIrVLC- and the BBIrVLC-TCM schemes in Figure 2.

By contrast, the corresponding EXIT-tunnel only becomes open for the SBVLC- and BBVLC-TCM bench-markers for $E_{b} / N_{0}$ values in excess of $3.7 \mathrm{~dB}$, which is $1.1 \mathrm{~dB}$ from the channel capacity bound of $2.6 \mathrm{~dB}$. We can therefore expect our SBIrVLC- and BBIrVLC-TCM schemes to be capable of operating at nearly half the distance from the channel capacity bound in comparison to our bench-markers, achieving a gain of about $0.5 \mathrm{~dB}$.

\section{RESULTS}

In this section, we discuss our findings when communicating over an uncorrelated narrowband Rayleigh fading channel having a range of $E_{b} / N_{0}$ values above the channel capacity bound of $2.6 \mathrm{~dB}$.

In addition to the proposed SBIrVLC-, BBIrVLC-, SBVLCand BBVLC-TCM schemes, in this section we also consider the operation of an additional bench-marker which we refer to as the Huffman-IrCC-TCM scheme. In contrast to the SBIrVLC-, BBIrVLC-, SBVLC- and BBVLC-TCM schemes, in the Huffman-IrCC-TCM scheme the transmission frame $\mathbf{u}$ of Figure 1 is generated by both Huffman and concatenated IrCC encoding the source symbol frame s, rather than by invoking VLC encoding. More specifically, Huffman coding is employed on a sub-frame by sub-frame basis, as described in Section II. The resultant frame of Huffman encoded bits $\mathbf{v}$ is protected by the memory-4 17-component IrCC scheme of [25]. This was tailored to have an overall coding rate of

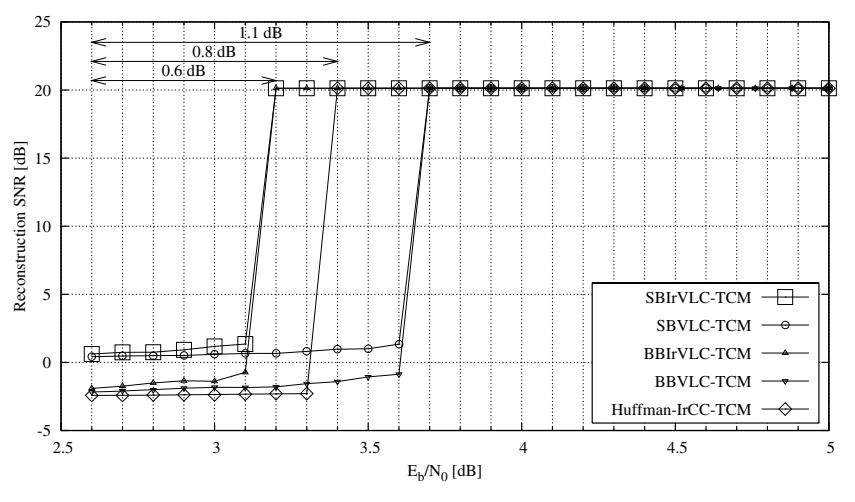

Fig. 3. Reconstruction SNR versus $E_{b} / N_{0}$ for a Gaussian source using $K=$ 16-level Lloyd-Max quantization for the SBIrVLC-, BBIrVLC-, SBVLCand BBVLC-TCM schemes, as well as for the Huffman-IrCC-TCM scheme, communicating over an uncorrelated narrowband Rayleigh fading channel following iterative decoding convergence.

0.52 and an inverted EXIT curve that does not cross the TCM EXIT curve at an $E_{b} / N_{0}$ of $3.2 \mathrm{~dB}$, just like the SBIrVLC and BBIrVLC designs detailed in Section III. In the Huffman-IrCC-TCM receiver, iterative APP SISO IrCC and TCM decoding proceeds, as described in Section II. Note that in addition to the a posteriori LLR frame $L_{p}^{o}(\mathbf{u})$ pertaining to the transmission frame $\mathbf{u}$, the APP SISO IrCC decoder can additionally provide the a posteriori LLR frame $L_{p}^{o}(\mathbf{v})$ pertaining to the frame of Huffman encoded bits $\mathbf{v}$. It is on the basis of this that bit-based MAP Huffman sequence estimation may be invoked on a sub-frame by sub-frame basis in order to obtain the source symbol frame estimate $\tilde{\mathbf{s}}$.

\section{A. Asymptotic performance following iterative decoding con- vergence}

For each of our schemes and for each value of $E_{b} / N_{0}$, we consider the reconstructed source sample frame $\tilde{\mathbf{e}}$ and evaluate the Signal to Noise Ratio (SNR) associated with the ratio of the source signal's energy and the reconstruction error energy that may be achieved following iterative decoding convergence. This relationship is plotted for each of the SBIrVLC-, BBIrVLC-, SBVLC- and BBVLC-TCM schemes, as well as for the Huffman-IrCC-TCM scheme, in Figure 3.

At sufficiently high $E_{b} / N_{0}$ values, all considered schemes are capable of achieving source sample reconstruction SNRs of up to $20 \mathrm{~dB}$, which represents the error-free case, where only quantization noise is present, while all channel-induced errors are absent. As shown in Figure 3, this may be achieved by the SBIrVLC- and BBIrVLC-TCM schemes at $E_{b} / N_{0}$ values above $3.2 \mathrm{~dB}$, which is just $0.6 \mathrm{~dB}$ from the channel capacity bound of $2.6 \mathrm{~dB}$. This represents a $0.5 \mathrm{~dB}$ gain over the SBVLC- and BBVLC-TCM schemes, which require $E_{b} / N_{0}$ values in excess of $3.7 \mathrm{~dB}$, a value that is $1.1 \mathrm{~dB}$ from the channel capacity bound. Additionally, the SBIrVLC- and BBIrVLC-TCM schemes can be seen to offer a $0.2 \mathrm{~dB}$ gain over the Huffman-IrCC-TCM scheme, which is incapable of operating within $0.8 \mathrm{~dB}$ of the channel capacity bound.

Note that our findings recorded in Figure 3 for the SBIrVLC-, BBIrVLC-, SBVLC- and BBVLC-TCM schemes confirm the EXIT chart predictions of Section III. Figure 2 provides decoding trajectories for the BBIrVLC-TCM and 
BBVLC-TCM schemes at $E_{b} / N_{0}$ values of $3.2 \mathrm{~dB}$ and 3.7 $\mathrm{dB}$, respectively. Note that owing to the sufficiently long interleaver length of $M \cdot J \cdot E / R=217,500$ bits, correlation within the a priori LLR frames $L_{a}^{i}(\mathbf{u})$ and $L_{a}^{o}(\mathbf{u})$ is mitigated and the recorded trajectories exhibit a close match with the corresponding TCM and inverted IrVLC/VLC EXIT curves. In both cases, the corresponding trajectory can be seen to converge to the $(1,1)$ mutual information point of the EXIT chart after a number of decoding iterations. Note that with each decoding iteration, a greater extrinsic mutual information is achieved, which corresponds to a greater source sample reconstruction SNR.

At low $E_{b} / N_{0}$ values, the corresponding TCM EXIT curves cross the inverted IrVLC or VLC EXIT curves and the open EXIT chart tunnel disappears. In these cases, iterative decoding convergence to unity mutual information cannot be achieved, resulting in the poor reconstruction quality that may be observed at low values of $E_{b} / N_{0}$ in Figure 3.

In the case of the Huffman-IrCC-TCM scheme however, poor reconstruction qualities were obtained despite the presence of an open EXIT chart tunnel for $E_{b} / N_{0}$ values between $3.2 \mathrm{~dB}$ and $3.4 \mathrm{~dB}$ in Figure 3. This surprising result may be attributed to the APP SISO IrCC decoder's relatively high sensitivity to any residual correlation within the a priori LLR frame $L_{a}^{o}(\mathbf{u})$ that could not be mitigated by the interleaver having an average length of 217,500 bits. As a result of this, the Huffman-IrCC-TCM EXIT trajectory does not approach the inverted IrCC EXIT curve very closely and a wide EXIT chart tunnel is required for iterative decoding convergence to the $(1,1)$ mutual information point of the EXIT chart.

The relatively high sensitivity of APP SISO CC decoding to any correlation within the a priori LLR frame $L_{a}^{o}(\mathbf{u})$ as compared to VLC decoding may be explained as follows. During APP SISO CC decoding using the BCJR algorithm, it is assumed that all a priori LLRs that correspond to bits within each set of $L$ consecutive codewords are uncorrelated, where $L$ is the constraint length of the CC [5]. By contrast, during APP SISO VLC decoding using the BCJR algorithm, it is assumed that all a priori LLRs that correspond to bits within each single codeword are uncorrelated [17]. Hence, the BCJRbased APP SISO decoding of a CC scheme can be expected to be more sensitive to correlation within the a priori LLR frame $L_{a}^{o}(\mathbf{u})$ than that of a VLC scheme having similar codeword lengths. As a result, a longer interleaver and hence a higher latency would be required for facilitating near-capacity $\mathrm{CC}$ operation.

\section{B. Performance during iterative decoding}

The achievement of iterative decoding convergence requires the completion of a sufficiently high number of decoding iterations. Clearly, each decoding iteration undertaken is associated with a particular computational complexity, the sum of which represents the total computational complexity of the iterative decoding process. Hence, the completion of a sufficiently high number of decoding iterations in order to achieve iterative decoding convergence may be associated with a high computational complexity. In order to quantify how this computational complexity scales as iterative decoding proceeds, we recorded the total number of ACS operations performed per source sample during APP SISO decoding and MAP sequence estimation.

Furthermore, the performance of the considered schemes was also assessed during the iterative decoding process, not only after its completion once convergence has been achieved. This was achieved by evaluating the source sample reconstruction SNR following the completion of each decoding iteration. The total computational complexity associated with this SNR was calculated as the sum of the computational complexities associated with all decoding iterations completed so far during the iterative decoding process. Clearly, as more and more decoding iterations are completed, the resultant source sample reconstruction SNR can be expected to increase until iterative decoding convergence is achieved. However, the associated total computational complexity will also increase as more and more decoding iterations are completed. Hence, this approach allows the characterisation of the tradeoff between reconstruction quality and computational complexity.

For each considered Rayleigh channel $E_{b} / N_{0}$ value, a set of source sample reconstruction SNRs and their corresponding computational complexities was obtained, as described above. Note that the size of these sets was equal to the number of decoding iterations required to achieve iterative decoding convergence at the particular $E_{b} / N_{0}$ value. It would therefore be possible to display the source sample reconstruction SNR versus both the $E_{b} / N_{0}$ and the computational complexity in a three-dimensional surface plot, for each of the SBIrVLC-, BBIrVLC-, SBVLC- and BBVLC-TCM schemes. For clarity however, these surfaces are projected in the direction of the source sample reconstruction SNR axis into two dimensions in Figure 4. We employ contours of constant source sample reconstruction SNR, namely $15 \mathrm{~dB}$ and $20 \mathrm{~dB}$, to parameterise the relationship between the Rayleigh fading channel's $E_{b} / N_{0}$ value and the associated computational complexity. Note that the plot of Figure 3 may be thought of as a cross-section through the surfaces represented by Figure 4, perpedicular to the computational complexity axis at $1 \cdot 10^{7}$ ACS operations per source sample. Note that this particular value of computational complexity is sufficiently high to achieve iterative decoding convergence at all values of $E_{b} / N_{0}$, in each of the considered schemes.

Note that the SBIrVLC and SBVLC decoders have a computational complexity per source sample that depends on the number of symbols in each source symbol sub-frame $\mathbf{s}^{\mathbf{m}}$, namely $J$. This is because the number of transitions in their symbol-based trellises is proportional to $J^{2}$ [15]. Hence the results provided in Figure 4 for the SBIrVLC- and SBVLCTCM schemes are specific to the $J=100$ scenario. By contrast, the TCM, BBIrVLC, BBVLC and IrCC decoders have a computational complexity per source sample that is independent of the number of symbols in each source symbol sub-frame $\mathbf{s}^{\mathbf{m}}$, namely $J$. This is because the number of transitions in their trellises is proportional to $J$ [14] [26] [5]. Hence the results for the BBIrVLC- and BBVLC-TCM schemes, as well as for the Huffman-IrCC-TCM scheme, provided in Figure 4 are not specific for the $J=100$ case.

As shown in Figure 4, source sample reconstruction SNRs of up to $20 \mathrm{~dB}$ can be achieved within $0.6 \mathrm{~dB}$ of the channel's 


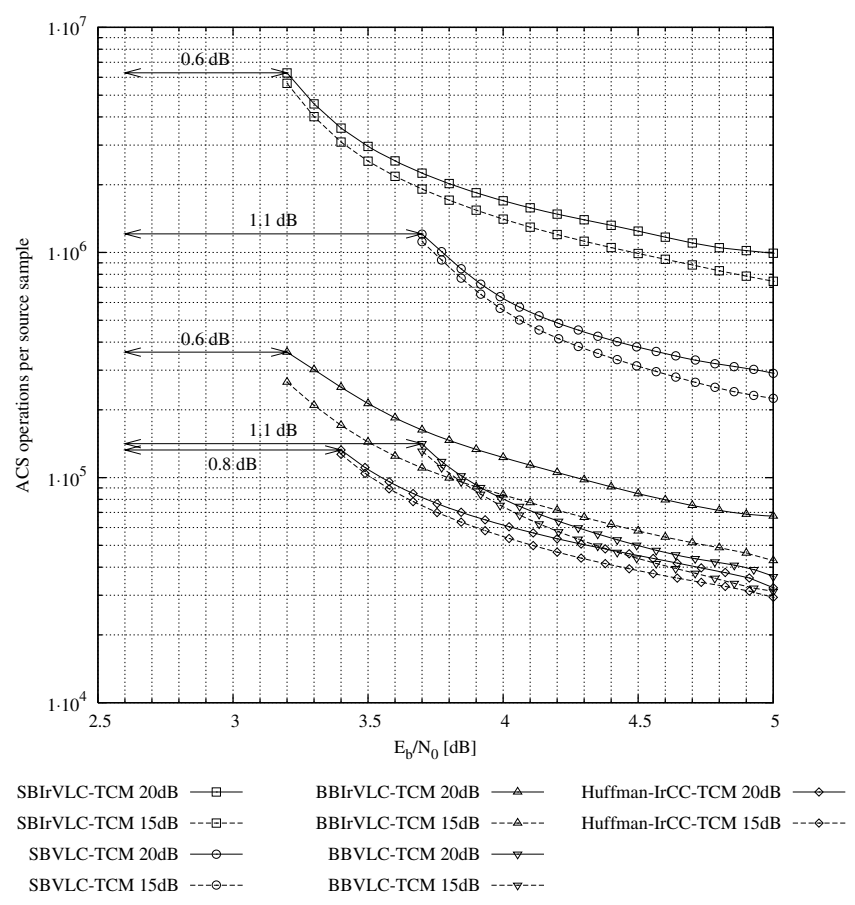

Fig. 4. Computational complexity versus $E_{b} / N_{0}$ for a Gaussian source using $K=16$-level Lloyd-Max quantization for the SBIrVLC-, BBIrVLC-, SBVLC- and BBVLC-TCM schemes, as well as for the Huffman-IrCC-TCM scheme, communicating over an uncorrelated narrowband Rayleigh fading channel, parameterised with the source sample reconstruction SNR.

capacity bound of $2.6 \mathrm{~dB}$ for the SBIrVLC- and BBIrVLCTCM schemes, within $1.1 \mathrm{~dB}$ for the SBVLC- and BBVLCTCM schemes and within $0.8 \mathrm{~dB}$ for the Huffman-IrCC-TCM scheme. Note that these findings agree with those of the EXIT chart analysis and the asymptotic performance analysis.

\section{Complexity analysis}

We now comment on the computational complexities of the considered schemes and select our preferred arrangement.

In all considered schemes and at all values of $E_{b} / N_{0}$, a source sample reconstruction SNR of $15 \mathrm{~dB}$ can be achieved at a lower computational complexity than an SNR of $20 \mathrm{~dB}$ can, as shown in Figure 4. This is because a reduced number of decoding iterations is required for achieving the extrinsic mutual information value associated with a lower reconstruction quality, as stated above. However, for all considered schemes operating at high values of $E_{b} / N_{0}$, this significant 5 $\mathrm{dB}$ reduction in source sample reconstruction SNR facilitates only a relatively modest reduction of the associated computational complexity, which was between $9 \%$ in the case of the Huffman-IrCC-TCM scheme and 36\% for the BBIrVLCTCM scheme. Hence we may conclude that the continuation of iterative decoding until near-perfect convergence is achieved can be justified at all values of $E_{b} / N_{0}$.

Additionally, it may be seen that a given source sample reconstruction SNR may be achieved at a reduced computational complexity for all considered schemes as the $E_{b} / N_{0}$ value increases. This may be explained by the widening of the EXIT chart tunnel, as the $E_{b} / N_{0}$ value increases. As a result, less decoding iterations are required for reaching the extrinsic mutual information that is associated with a specific source sample reconstruction SNR considered.

In each of the considered schemes it was found that VLC and $\mathrm{CC}$ decoding is associated with a higher contribution to the total computational complexity than TCM decoding. Indeed, in the case of the SBIrVLC- and SBVLC-TCM schemes, it was found that VLC decoding accounts for about $97 \%$ of the numbers of ACS operations per source sample, having a complexity of about 32.3 times higher than that of TCM decoding. By contrast, in the BBIrVLC- and BBVLCTCM schemes, VLC decoding accounts for only $70 \%$ of the operations, having a complexity of about 2.3 times that of TCM decoding. Similarly, CC decoding accounts for only $60 \%$ of the ACS operations in the Huffman-IrCC-TCM scheme, having a complexity of about 1.4 times that of TCM decoding.

The high complexity of the SBIrVLC and SBVLC decoders may be attributed to the specific structure of their trellises, which contain significantly more transitions than those of the BBIrVLC, BBVLC and IrCC decoders [15]. As a result, the SBIrVLC- and SBVLC-TCM schemes have a complexity that is about an order of magnitude higher than that of the BBIrVLC- and BBVLC-TCM schemes, as well as the Huffman-IrCC-TCM scheme, as shown in Figure 4. In the light of this, the employment of the SBIrVLC- and SBVLCTCM schemes cannot be readily justified.

Observe in Figure 4 that at high $E_{b} / N_{0}$ values, the SBIrVLC- and BBIrVLC-TCM schemes have a higher computational complexity than the corresponding SBVLC- or BBVLC-TCM scheme. This is due to the influence of their low rate VLC codebook components. These codebooks comprise codewords with many different lengths, which introduce many transitions, when represented in a trellis structure. The observed computational complexity discrepancy is particularly high in the case of the schemes that employ the symbol-based VLC trellis, owing to its particular nature. For this reason, the SBIrVLC-TCM scheme has a computational complexity that is $240 \%$ higher than that of the SBVLC-TCM scheme.

By contrast, we note that at high values of $E_{b} / N_{0}$ the BBIrVLC-TCM scheme has only about a $60 \%$ higher computational complexity than the BBVLC-TCM scheme. Similarly, the BBIrVLC-TCM scheme has only twice the computational complexity of the Huffman-IrCC-TCM scheme. Coupled with the BBIrVLC-TCM scheme's ability to operate within $0.6 \mathrm{~dB}$ of the Rayleigh fading channel's capacity bound, we are able to identify this as our preferred arrangement.

\section{CONClusion}

In this paper we have introduced a novel IrVLC design for near-capacity joint source and channel coding. In analogy to IrCC, IrVLC employs a number of component VLC codebooks having different coding rates in appropriate proportions. More specifically, with the aid of EXIT chart analysis, the appropriate fractions of the input source symbols may be chosen for directly ensuring that the EXIT curve of the IrVLC codec may be matched to that of a serially concatenated channel codec. In this way, an open EXIT chart tunnel facilitating near-capacity high quality source sample reconstruction may be achieved. 
We have detailed the construction of an IrVLC scheme that is suitable for the encoding of 16-level Lloyd-Max quantized Gaussian i.i.d. source samples and for use with IQ-interleaved TCM and 16QAM over uncorrelated narrowband Rayleigh fading channels. For the purposes of comparison, we also selected a regular VLC bench-marker, having a coding rate equal to that of our IrVLC scheme. Serially-concatenated and iteratively decoded SBIrVLC-, BBIrVLC-, SBVLC- and BBVLC-TCM schemes were characterised with the aid of EXIT chart analysis. These schemes have a bandwidth efficiency of 1.56 bits per channel symbol, which corresponds to a Rayleigh fading channel capacity bound of $2.6 \mathrm{~dB}$. Using an average interleaver length of 217,500 bits, the SBIrVLC- and BBIrVLC-TCM schemes were found to offer high-quality source sample reconstruction at an $E_{b} / N_{0}$ value of $3.2 \mathrm{~dB}$, which is just $0.6 \mathrm{~dB}$ from the capacity bound. This compares favourably with the SBVLC- and BBVLCTCM bench-markers, which require an $E_{b} / N_{0}$ value of 3.7 dB. This also compares favourably with a Huffman-IrCCTCM bench-marker, which requires an $E_{b} / N_{0}$ value of 3.4 $\mathrm{dB}$ owing to its slightly eroded performance when operating with the considered interleaver length. Owing to the higher computational complexity of the SBIrVLC-TCM scheme, the BBIrVLC-TCM arrangement was identified as our preferred scheme.

\section{REFERENCES}

[1] M. Tüchler and J. Hagenauer, "EXIT charts of irregular codes," in Proc. Conference on Information Sciences and Systems, Princeton, NJ, Mar. 2002, pp. 748-753.

[2] S. ten Brink, "Convergence of iterative decoding," Electron. Lett., vol. 35 , no. 10 , pp. 806-808, 1999.

[3] A. Ashikhmin, G. Kramer, and S. ten Brink, "Extrinsic information transfer functions: model and erasure channel properties," IEEE Trans. Inform. Theory, vol. 50, no. 11, pp. 2657-2673, Nov. 2004.

[4] J. Wang, S. X. Ng, A. Wolfgang, L.-L. Yang, S. Chen, and L. Hanzo, "Near-capacity three-stage MMSE turbo equalization using irregular convolutional codes," in International Symposium on Turbo Codes, Munich, Germany, Apr. 2006, electronic publication.

[5] L. Hanzo, T. H. Liew, and B. L. Yeap, Turbo Coding, Turbo Equalisation and Space Time Coding for Transmission over Wireless Channels. Chichester, UK: Wiley, 2002.

[6] L. Hanzo, F. C. A. Somerville, and J. P. Woodard, Voice and Audio Compression for Wireless Communications. John Wiley and IEEE Press, to appear.

[7] L. Hanzo, P. J. Cherriman, and J. Streit, Video Compression and Communications: H.261, H.263, H.264, MPEG4 and Proprietary Codecs for HSDPA-Style Adaptive Turbo-Transceivers. John Wiley and IEEE Press, to appear.

[8] C. E. Shannon, "The mathematical theory of communication," Bell Syst. Techn. J., vol. 27, pp. 379-423, July 1948.

[9] D. A. Huffman, "A method for the construction of minimum-redundancy codes," Proc. IRE, vol. 40, pp. 1098-1101, Sept. 1952.

[10] V. Buttigieg and P. G. Farrell, "Variable-length error-correcting codes," IEE Proc. Commun., vol. 147, no. 4, pp. 211-215, Aug. 2000.

[11] S. Benedetto and G. Montorsi, "Serial concatenation of block and convolutional codes," Electron. Lett., vol. 32, no. 10, pp. 887-888, 1996.

[12] — , "Iterative decoding of serially concatenated convolutional codes," Electron. Lett., vol. 32, no. 13, pp. 1186-1188, 1996.

[13] R. G. Maunder, J. Wang, S. X. Ng, L.-L. Yang, and L. Hanzo, "Iteratively Decoded Irregular Variable Length Coding and Trellis Coded Modulation," submitted to IEEE Workshop on Signal Processing Systems, Shanghai, China, October 2007. [Online]. Available: http://www.ecs.soton.ac.uk/people/rm02r

[14] G. Ungerboeck, "Channel coding with multilevel/phase signals," IEEE Trans. Inform. Theory, vol. 28, no. 1, pp. 55-67, 1982.

[15] R. Bauer and J. Hagenauer, "Symbol by symbol MAP decoding of variable length codes," in Proc. 3rd ITG Conference on Source and Channel Coding, Munich, Germany, Jan. 2000, pp. 111-116.
[16] J. Kliewer and R. Thobaben, "Iterative joint source-channel decoding of variable-length codes using residual source redundancy," IEEE Trans. Wireless Commun., vol. 4, no. 3, pp. 919-929, 2005.

[17] V. B. Balakirsky, "Joint source-channel coding with variable length codes," in Proc. IEEE International Symposium on Information Theory, Ulm, Germany, June 1997, p. 419.

[18] S. Lloyd, "Least squares quantization in PCM," IEEE Trans. Inform. Theory, vol. 28, no. 2, pp. 129-137, 1982.

[19] J. Max, "Quantizing for minimum distortion," IRE Trans. Inform. Theory, vol. 6, no. 1, pp. 7-12, Mar. 1960.

[20] L. Bahl, J. Cocke, F. Jelinek, and J. Raviv, "Optimal decoding of linear codes for minimizing symbol error rate (Corresp.)," IEEE Trans. Inform. Theory, vol. 20, no. 2, pp. 284-287, 1974.

[21] J. Hagenauer, E. Offer, and L. Papke, "Iterative decoding of binary block and convolutional codes," IEEE Trans. Inform. Theory, vol. 42, no. 2 , pp. 429-445, 1996.

[22] J. Wang, L.-L. Yang, and L. Hanzo, "Iterative construction of reversible variable-length codes and variable-length error-correcting codes," IEEE Commun. Lett., vol. 8, no. 11, pp. 671-673, Nov. 2004.

[23] R. Thobaben and J. Kliewer, "Low-complexity iterative joint sourcechannel decoding for variable-length encoded Markov sources," IEEE Trans. Commun., vol. 53, no. 12, pp. 2054-2064, Dec. 2005.

[24] L. Hanzo, S. X. Ng, T. Keller, and W. Webb, Quadrature Amplitude Modulation. Chichester, UK: Wiley, 2004.

[25] M. Tüchler, "Design of serially concatenated systems depending on the block length," IEEE Trans. Commun., vol. 52, no. 2, pp. 209-218, Feb. 2004.

[26] R. Bauer and J. Hagenauer, "On variable length codes for iterative source/channel decoding," in Proc. Data Compression Conference, Snowbird, UT, Mar. 2001, pp. 273-282.

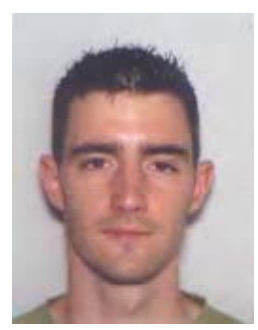

Robert G. Maunder has studied with the School of Electronics and Computer Science, University of Southampton, UK, since October 2000. He was awarded a first class honours BEng in Electronic Engineering in July 2003, shortly before beginning his current $\mathrm{PhD}$ studies in the Communications Research Group at the same university. His research interests include video coding, joint source/channel coding and iterative decoding. He has published a number of IEEE papers in these areas.

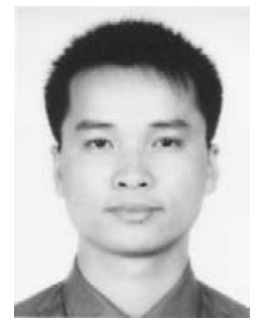

Jin Wang (S'03) received the B.E. degree from University of Science and Technology of China (USTC), Hefei, China in 1999 and the M.E. degree in video signal processing from Graduate School of Chinese Academy of Sciences (GSCAS), Beijing, China, in 2002. He is currently pursuing his $\mathrm{PhD}$ degree with the Communications Research Group at the School of ECS, University of Southampton, Southampton, UK. His research interests include video coding, channel coding, joint source/channel coding, and iterative detection and decoding for digital communication systems.

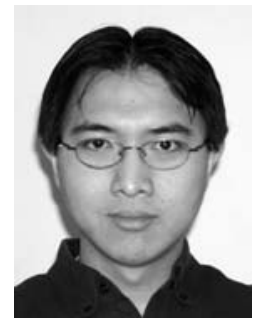

Soon Xin Ng ( $\mathrm{S}^{\prime} 99-\mathrm{M}^{\prime} 03$ ) received the B.Eng. degree (First class) in electronics engineering and the Ph.D. degree in wireless communications from the University of Southampton, Southampton, U.K., in 1999 and 2002, respectively. From 2003 to 2006, he was a postdoctoral research fellow working on collaborative European research projects known as SCOUT, NEWCOM and PHOENIX. Since August 2006, he has been a lecturer in wireless communications at the University of Southampton. His research interests are mainly in adaptive coded modulation, channel coding, space-time coding, joint source and channel coding, OFDM and MIMO. He has published numerous papers and coauthored a book in this field. 


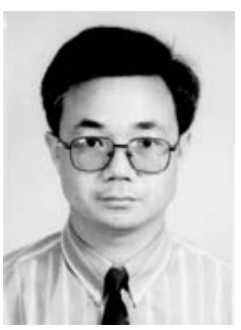

Dr. Lie-Liang Yang received his B.Eng. degree in communication engineering from Shanghai TieDao University, Shanghai, China in 1988, and his M.Eng, Ph.D. degrees in communications and electronics from Northern Jiaotong University, Beijing, China in 1991 and 1997, respectively. From June 1997 to December 1997 he was a visiting scientist of the Institute of Radio Engineering and Electronics, Academy of Sciences of the Czech Republic. Since December 1997, he has been with the Communications Research Group, School of Electronics and Computer Science, University of Southampton, U.K, where he was first a Postdoctoral Research Fellow (Dec. 1997 - Aug. 2002), then a Lecturer (Sept. 2002 - Feb. 2006), and currently holds the academic post of Readership. Dr. Yang's research has covered a wide range of areas in telecommunications, which include error control coding, modulation and demodulation, spreadspectrum communications and multiuser detection, synchronization, spacetime processing, adaptive wireless systems, as well as wideband, broadband and ultrawide-band code-division multiple-access (CDMA). He has published over 130 papers in journals and conference proceedings, coauthored one book and published several book chapters. He was awarded the Royal Society SinoBritish Fellowship in 1997 and the EPSRC Research Fellowship in 1998. Dr. Yang is currently an associate editor for both the Journal of Communications and Networks (JCN) and the Journal of Communications (JCM).

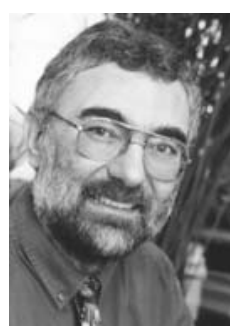

Lajos Hanzo, Fellow of the Royal Academy of Engineering, received his first-class degree in electronics in 1976 and his doctorate in 1983. In 2004 he was awarded the Doctor of Sciences (DSc) degree by the University of Southampton, UK. During his career in telecommunications he has held various research and academic posts in Hungary, Germany and the UK. Since 1986 he has been with the Department of Electronics and Computer Science, University of Southampton, UK, where he holds the chair in telecommunications. He has co-authored 15 books, totalling 10000 pages on mobile radio communications, published in excess of 700 research papers, has acted as TPC Chair of numerous major IEE and IEEE conferences, presented various keynote lectures and has been awarded a number of distinctions. Currently he heads an academic research team, working on a range of research projects in the field of wireless multimedia communications sponsored by industry, the Engineering and Physical Sciences Research Council (EPSRC) UK, the European IST Programme and the Mobile Virtual Centre of Excellence (VCE), UK. He is an enthusiastic supporter of industrial and academic liaison and he offers a range of industrial courses. He is also an IEEE Distinguished Lecturer of both the Communications as well as the Vehicular Technology Society, a Fellow of both the IEEE and the IEE. He is an editorial board member of the Proceedings of the IEEE and a Governor of the IEEE VT Society. For further information on research in progress and associated publications, please refer to http://www-mobile.ecs.soton.ac.uk 